\title{
Robust RLS Wiener FIR Filter for Signal Estimation in Linear Discrete-Time Stochastic Systems with Uncertain Parameters
}

\author{
Seiichi Nakamori \\ Department of Technology, Faculty of Education, Kagoshima University, Kagoshima, Japan \\ Email: k6165161@kadai.jp
}

\begin{abstract}
This paper proposes the robust recursive least-squares (RLS) finite impulse response (FIR) filtering algorithm using the covariance information and the robust RLS Wiener FIR filtering algorithm in linear discrete-time stochastic systems with the parameter uncertainties. The observation and system matrices contain the uncertain parameters. The uncertain parameters cause the degraded signal. Theorem 2 proposes the robust RLS FIR filter using the covariance information of the state vector for the degraded signal, the cross-covariance information of the state vector for the signal with the state vector for the degraded signal, the observation matrices for the signal and the degraded signal, and the variance of the white observation noise. Here, it is assumed that the signal and the degraded signal are fitted to the finite-order autoregressive (AR) models. Theorem 3 proposes the robust RLS Wiener FIR filter. The robust RLS Wiener FIR filtering algorithm uses the system and observation matrices for the signal and the degraded signal, the variance of the state vector for the degraded signal, the cross-variance function of the state vector for the signal with the state vector for the degraded signal, and the variance of the white observation noise.
\end{abstract}

Keywords: Robust RLS Wiener FIR filter; covariance information; Wiener- Hopf equation; uncertain parameters; degraded signal.

\section{Introduction}

In comparison with the Kalman filter having the infinite impulse response (IIR) property, the maximumlikelihood filter [1] with recent finite data is less sensitive to dynamical model errors. The finite impulse response (FIR) Kalman filter [2]-[5] has robustness property. In [6], the FIR filter for visual object tracking is proposed by maximizing the likelihood function with superior tracking results in comparison with the Kalman filter, the particle filter and the $H_{\infty}$ filter. In [7], the robust weighted fusion Kalman estimators are presented for multi-sensor systems with the multiplicative noises, missing measurements, and the white noise correlated in the input and the observation. It is assumed that $\phi_{k}(t) 1 \leq k \leq q$, multiplied by the multiplicative noise in the state equation, are known.

Recently, the robust recursive least-squares (RLS) Wiener fixed-point smoother and filter, and the robust RLS estimators using the covariance information [8] are designed for the linear discrete-time stochastic systems with the uncertain parameters in the system and observation matrices. In [9], the robust Kalman filter is proposed in linear discrete-time stochastic systems with the uncertain parameters characterized by the norm-bounded condition [9]. In [10], the RLS Wiener FIR predictor and filter calculate the least-squares estimates by using the accurate information on the system matrix, the observation matrix and the variance of the state vector. As is clear from the simulation result in section 5 , the drawback of the robust Kalman filter [9] and the RLS Wiener filter in [10] lie in the point that they compute the estimates with the degraded estimation accuracies, given the uncertain information on the system and observation matrices. To compensate this deficiency, this paper develops the robust RLS Wiener FIR filter based on the innovation theory in linear discrete-time stochastic systems. As the finite interval $L$ increases, the MSV of the filtering errors by the robust RLS Wiener filter FIR decreases and approaches the MSV by the robust RLS Wiener filter [8] for each observation noise. This indicates, with the finite number of observations, that the robust RLS Wiener FIR filter in Theorem 3 could attain almost the same estimation accuracy with the robust RLS Wiener filter [8]. In addition, the robust RLS FIR filter using the covariance information is proposed in this paper. Similarly to the robust RLS Wiener estimators [8], the robust RLS Wiener FIR filter and the robust RLS FIR filter using the covariance information do 
not use the norm-bounded condition [9] posed on the uncertain parameters. It is assumed that the signal process is fitted to the auto-regressive (AR) model of the finite order. Also, the degraded signal, caused by the uncertain parameters in the observation and system matrices, is fitted to the AR model of the finite order. From the AR model, the system and observation matrices are obtained in the state-space model for the degraded signal. Based on the problem formulation on the robust least-squares FIR filtering problem, the Wiener-Hopf equation is obtained. From the Wiener-Hopf equation, the robust RLS FIR filtering algorithm using the covariance information and the robust RLS Wiener FIR filtering algorithm are derived by the invariant imbedding method. The robust RLS Wiener FIR filtering algorithm uses the system and observation matrices for the signal and the degraded signal, the variance of the state vector for the degraded signal, the cross-variance function of the state vector for the signal with the state vector for the degraded signal, and the variance of the white observation noise. Also, the robust RLS FIR filter uses the covariance information of the state vector for the degraded signal, the cross-covariance information of the state vector for the signal with the state vector for the degraded signal, the observation matrices for the signal and the degraded signal besides the variance of the white observation noise.

In a numerical simulation example, the estimation accuracy of the robust RLS Wiener FIR filter, proposed in this paper, is compared with the robust RLS Wiener filter [8], the robust Kalman filter [9] and the RLS Wiener FIR filter [10].

\section{Robust Least-Squares FIR Filtering Problem}

Let an m-dimensional observation equation and an n-dimensional state equation be described by

$$
\begin{aligned}
& \breve{y}(k)=\breve{z}(k)+v(k), \breve{z}(k)=\bar{H}(k) \bar{x}(k), \bar{H}(k)=H+\Delta H(k), \\
& \bar{x}(k+1)=\bar{\Phi}(k) \bar{x}(k)+\Gamma w(k), \bar{\Phi}(k)=\Phi+\Delta \Phi(k), \\
& E\left[v(k) v^{T}(s)\right]=R \delta_{K}(k-s), E\left[w(k) w^{T}(s)\right]=Q \delta_{K}(k-s),
\end{aligned}
$$

in linear discrete-time stochastic systems with the uncertain parameters [8]. $\Delta H(k)$ and $\Delta \Phi(k)$ represent the uncertain parameters. $v(k)$ denotes the white observation noise with the variance $R$ and $w(k)$ is the white input noise with the variance $Q$. Their auto-covariance functions are given in (1) using the Kronecker delta function $\delta_{K}(k-s)$. The state equation generating $\bar{x}(k+1)$ includes the uncertain quantity $\Delta \Phi(k)$ in the system matrix $\bar{\Phi}(k)$. Similarly, in the observation equation the observation matrix $\bar{H}(k)$ contains the uncertain quantity $\Delta H(k)$. Hence, $\breve{z}(k)$ is the degraded value against the nominal signal $z(k)$ generated by the state-space model (2), which does not include the uncertain quantities. In (1), as the sum of the degraded signal $\breve{z}(k)$ and the observation noise $v(k), \breve{y}(k)$ is measured. The state-space model without containing the uncertain quantities $\Delta H(k)$ and $\Delta \Phi(k)$ in (1) is expressed by

$$
\begin{aligned}
& y(k)=z(k)+v(k), z(k)=H x(k), \\
& x(k+1)=\Phi x(k)+\Gamma w(k) .
\end{aligned}
$$

In $(2), z(k)$ is the signal to be estimated. $H$ is an $m$ by $n$ observation matrix, $x(k)$ is the state vector and $v(k)$ is the white observation noise with the auto-covariance function in (1). The auto-covariance function of the input noise $w(k)$ is also given in (1). It is assumed that the processes of the signal and the observation noise are mutually independent and have zero means. This paper, based on the innovation theory, designs the robust RLS FIR filter using the covariance information and the robust RLS Wiener FIR filter estimating the signal $z(k)$ with the measurement data $\breve{y}(k)$ without any knowledge of the uncertain quantities $\Delta \Phi(k)$ and $\Delta H(k)$.

It is assumed that the sequence of the degraded signal $\breve{z}(k)$ is fitted to the AR model of the order $N$.

$$
\begin{aligned}
& \breve{z}(k)=-a_{1} \breve{z}(k-1)-a_{2} \breve{z}(k-2) \cdots-a_{N} \breve{z}(k-N)+\breve{e}(k), \\
& E\left[\breve{e}(k) \breve{e}^{T}(s)\right]=\breve{Q} \delta_{K}(k-s)
\end{aligned}
$$


Let $\breve{z}(k)$ be expressed with the state vector $\breve{x}(k)$ by

$$
\begin{aligned}
& \breve{z}(k)=\breve{H} \breve{x}(k), \\
& \breve{x}(k)=\left[\begin{array}{c}
\breve{x}_{1}(k) \\
\breve{x}_{2}(k) \\
\vdots \\
\breve{x}_{N-1}(k) \\
\breve{x}_{N}(k)
\end{array}\right]=\left[\begin{array}{c}
\breve{z}(k) \\
\breve{z}(k+1) \\
\vdots \\
\breve{z}(k+N-2) \\
\breve{z}(k+N-1)
\end{array}\right],
\end{aligned}
$$

Accordingly, the state equation for the state vector $\breve{x}(k)$ is given by

$$
\begin{aligned}
& {\left[\begin{array}{c}
\breve{x}_{1}(k+1) \\
\breve{x}_{2}(k+1) \\
\vdots \\
\breve{x}_{N-1}(k+1) \\
\breve{x}_{N}(k+1)
\end{array}\right]=\left[\begin{array}{ccccc}
0 & I_{m \times m} & 0 & \cdots & 0 \\
0 & 0 & I_{m \times m} & \cdots & 0 \\
\vdots & \vdots & \vdots & \ddots & \vdots \\
0 & 0 & 0 & \cdots & I_{m \times m} \\
-\breve{a}_{N}-\breve{a}_{N-1} & -\breve{a}_{N-2} \cdots & -\breve{a}_{1}
\end{array}\right]\left[\begin{array}{c}
\breve{x}_{1}(k) \\
\breve{x}_{2}(k) \\
\vdots \\
\breve{x}_{N-1}(k) \\
\breve{x}_{N}(k)
\end{array}\right]+\left[\begin{array}{c}
0 \\
0 \\
\vdots \\
0 \\
I_{m \times m}
\end{array}\right] \zeta(k),} \\
& \zeta(k)=\breve{e}(k+N), E\left[\zeta(k) \zeta^{T}(s)\right]=\breve{Q} \delta_{K}(k-s) .
\end{aligned}
$$

Let $\breve{K}(k, s)=\breve{K}(k-s)$ represent the auto-covariance function of the state vector $\breve{x}(k)$ in wide-sense stationary stochastic systems [11], and let $\breve{K}(k, s)$ be expressed in the form of the semi-degenerate kernel

$$
\begin{aligned}
& \breve{K}(k, s)=\left\{\begin{array}{l}
A(k) B^{T}(s), 0 \leq s \leq k, \\
B(k) A^{T}(s), 0 \leq k \leq s,
\end{array}\right. \\
& A(k)=\breve{\Phi}^{k}, B^{T}(s)=\breve{\Phi}^{-s} \breve{K}(s, s)
\end{aligned}
$$

Here, $\breve{\Phi}$ denotes the system matrix for the state vector $\breve{x}(k)$. From the state equation (5), the system matrix $\breve{\Phi}$ is given by

$$
\breve{\Phi}=\left[\begin{array}{ccccc}
0 & I_{m \times m} & 0 & \cdots & 0 \\
0 & 0 & I_{m \times m} & \cdots & 0 \\
\vdots & \vdots & \vdots & \ddots & \vdots \\
0 & 0 & 0 & \cdots & I_{m \times m} \\
-\breve{a}_{N} & -\breve{a}_{N-1} & -\breve{a}_{N-2} & \cdots & -\breve{a}_{1}
\end{array}\right]
$$

Also, by putting $K_{\breve{z}}(k, s)=K_{\breve{z}}(k-s)=E\left[\breve{z}(k) \breve{z}^{T}(s)\right]$, the auto-variance function $\breve{K}(k, k)$ of the state vector $\breve{x}(k)$ is described by

$$
\begin{aligned}
& \breve{K}(k, k)=E\left[\left[\begin{array}{c}
\breve{z}(k) \\
\breve{z}(k+1) \\
\vdots \\
\breve{z}(k+N-2) \\
\breve{z}(k+N-1)
\end{array}\right]\right. \\
& \left.\times\left[\breve{z}^{T}(k) \breve{z}^{T}(k+1) \cdots \breve{z}^{T}(k+N-2) \breve{z}^{T}(k+N-1)\right]\right] \\
& =\left[\begin{array}{ccccc}
K_{\breve{z}}(0) & K_{\breve{z}}(-1) & \cdots & K_{\breve{z}}(-N+2) & K_{\breve{z}}(-N+1) \\
K_{\breve{z}}(1) & K_{\breve{z}(0)} & \cdots & K_{\breve{z}}(-N+3) & K_{\breve{z}}(-N+2) \\
\vdots & \vdots & \ddots & \vdots & \vdots \\
K_{\breve{z}}(N-2) & K_{\breve{z}}(N-3) & \cdots & K_{\breve{z}}(0) & K_{\breve{z}(-1)} \\
K_{\bar{z}}(N-1) & K_{\bar{z}}(N-2) & \cdots & K_{\breve{z}}(1) & K_{\breve{z}}(0)
\end{array}\right] .
\end{aligned}
$$

By using $K_{z}(k-s)$, the Yule-Walker equation for the AR parameters is given by 


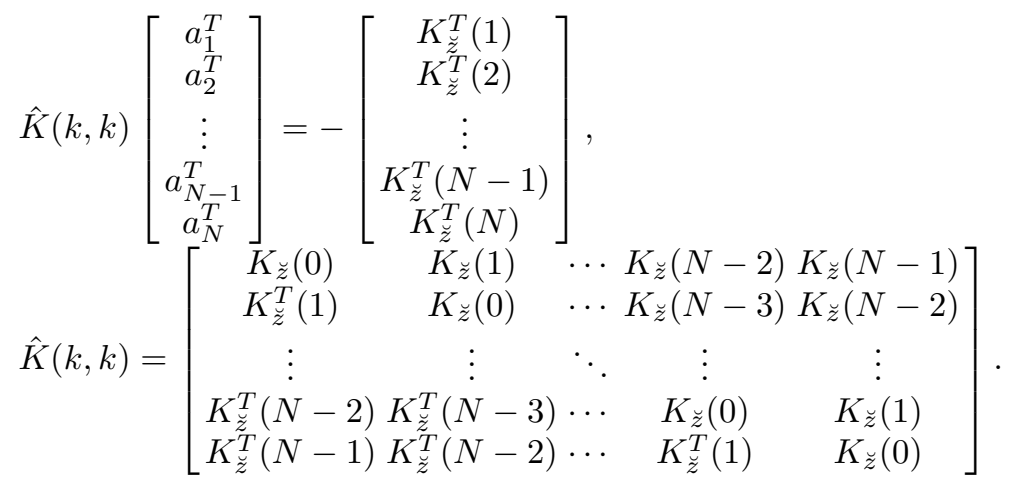

Let $K_{x \breve{x}}(k, s)=K_{x \breve{x}}(k-s)=E\left[x(k) \breve{x}^{T}(s)\right]$ represent the cross-covariance function of the state vector $x(k)$ with $\breve{x}(s)$ in wide-sense stationary stochastic systems. Let $K_{x \breve{x}}(k, s)$ be expressed in the form of

$$
\begin{aligned}
& K_{x \breve{x}}(k, s)=\alpha(k) \beta^{T}(s), 0 \leq s \leq k, \\
& \alpha(k)=\Phi^{k}, \beta^{T}(s)=\Phi^{-s} K_{x \breve{x}}(s, s) .
\end{aligned}
$$

From (2), $\Phi$ denotes the system matrix for the state vector $x(k)$.

Theorem 1 Let the FIR filtering estimate $\hat{x}(k \mid k-L+1)$ of the state vector $x(k)$ be expressed by

$$
\begin{aligned}
& \hat{x}(k \mid k-L+1)=\sum_{i=k-L+1}^{k} g(k, i) v(i), \\
& v(i)=\breve{y}(i)-\breve{H} \breve{\Phi} \hat{x}(i-1 \mid i-1-L+1),
\end{aligned}
$$

in terms of the innovation process $\{v(i), k-L+1 \leq i \leq k\}$. In (11), $g(k, i)$ represents a time-varying impulse response function and $\hat{x}(i-1 \mid i-1-L+1)$ is the FIR filtering estimate of the state vector $\breve{x}(i-1)$. Then the optimal impulse response function $g(k, s)$ satisfies

$$
\begin{aligned}
& g(k, s) \Lambda(s)=K_{x \breve{x}}(k, s) \breve{H}^{T}-\sum_{i=s-1-L+1}^{s-1} g(k, i) \Lambda(i) g^{T}(s-1, i) \breve{\Phi}^{T} \breve{H}^{T}, \\
& K_{x \breve{x}}(k, s) \breve{H}^{T}=K_{x \breve{z}}(k, s) .
\end{aligned}
$$

In (12), $K_{x \breve{x}}(k, s) \breve{H}^{T}$ equals the cross-covariance function of the state vector $x(k)$ with the degraded signal $\breve{z}(s), K_{x \breve{z}}(k, s)$.

\section{Proof}

Let us consider the estimation problem, which minimizes the mean-square value (MSV)

$$
J=E\left[\|x(k)-\hat{x}(k \mid k-L+1)\|^{2}\right]
$$

of the FIR filtering errors. From an orthogonal projection lemma [11]

$$
x(k)-\sum_{i=k-L+1}^{k} g(k, i) v(i) \perp v(s), k-L+1 \leq s \leq k,
$$

the impulse response function $g(k, i)$ satisfies the Wiener-Hopf equation

$$
E\left[x(k) v^{T}(s)\right]=\sum_{i=k-L+1}^{k} g(k, i) E\left[v(i) v^{T}(s)\right], k-L+1 \leq s \leq k .
$$

In (14), ' $\perp$ ' denotes the notation of the orthogonality. Let the covariance function of the innovation process satisfy

$$
E\left[v(i) v^{T}(s)\right]=\Lambda(i) \delta_{K}(i-s) .
$$


From $(15),(16)$ and the expression for the innovation process $v(s)$, it follows that

$$
\begin{aligned}
& g(k, s) \Lambda(s)=E\left[x(k) v^{T}(s)\right] \\
& =E\left[x(k)(\breve{y}(s)-\breve{H} \breve{\Phi v}(s-1 \mid s-1-L+1))^{T}\right] \\
& =E\left[x(k) \breve{y}^{T}(s)\right]-E\left[x(k) v^{T}(s-1 \mid s-1-L+1)\right] \breve{\Phi}^{T} \breve{H}^{T} .
\end{aligned}
$$

The term $E\left[x(k) \breve{y}^{T}(s)\right]$ in (17) is developed as follows.

$$
\begin{aligned}
& E\left[x(k) \breve{y}^{T}(s)\right] \\
& =E\left[x(k)(\breve{z}(s)+v(s))^{T}\right] \\
& =E\left[x(k) \breve{z}^{T}(s)\right] \\
& =E\left[x(k) \breve{x}^{T}(s)\right] \breve{H}^{T} \\
& =K_{x \breve{x}}(k, s) \breve{H}^{T} .
\end{aligned}
$$

From (17) and (18), the optimal impulse response function $g(k, s)$ satisfies (12).

(Q.E.D.)

Based on the preliminary formulation on the RLS FIR filtering problems, the robust RLS FIR filter using the covariance information and the robust RLS Wiener FIR filter are presented in section 3.

\section{Robust RLS FIR Filter Using Covariance Information and Robust RLS Wiener FIR Filter}

Theorem 2 presents the robust RLS FIR filtering algorithm using the covariance information $\breve{K}(k, k)$ of the state vector $\breve{x}(k)$ for the degraded signal $\breve{z}(k)$ and the cross-variance information $K_{x \breve{x}}(k, k)$ of the state vector $x(k)$ for the signal $z(k)$ with the state vector $\breve{x}(k)$ for the degraded signal $\breve{z}(k)$ in the state-space model (1) including the uncertain parameters.

Theorem 2 Let the state equation and the observation equation, including the uncertain quantities $\Delta \Phi$ and $\Delta H$ respectively, be given by (1). Let $\Phi$ and $H$ represent the system and observation matrices respectively for the signal $z(k)$. Let $\breve{\Phi}$ and $\breve{H}$ represent the system and observation matrices respectively for the degraded signal $\breve{z}(k)$, which is fitted to the AR model $(3)$ of the order $N$. Let the variance $\breve{K}(k, k)$ of the state vector $\breve{x}(k)$ for the degraded signal $\breve{z}(k)$ and the cross-variance function $K_{x \breve{x}}(k, k)$ of the state vector $x(k)$ for the signal $z(k)$ with $\breve{x}(k)$ be given. Let the auto-covariance function of $\breve{x}(k)$ be expressed by (6) in terms of $A(k)$ and $B(s)$. Let the cross-covariance function of $x(k)$ with $\breve{x}(s)$ be given by (10) in terms of $\alpha(k)$ and $\beta(s)$. Let the variance of the white observation noise $v(k)$ be $R$. Then, the robust RLS algorithm for the FIR filtering estimate $\hat{z}(k \mid k-L+1)$ of the signal $z(k)$ consists of (19)-(36) in linear discrete-time stochastic systems.

FIR filtering estimate of the signal $z(k): \hat{z}(k \mid k-L+1)$

$$
\hat{z}(k \mid k-L+1)=H \hat{x}(k \mid k-L+1)
$$

FIR filtering estimate of the state vector $x(k): \hat{x}(k \mid k-L+1)$

$$
\hat{x}(k \mid k-L+1)=\alpha(k) e(k)
$$

Initial condition of $\hat{x}(k \mid k-L+1)$ at $k=L: \hat{x}(L \mid 1)$

FIR filtering estimate of the state vector $\breve{x}(k): \hat{x}(k \mid k-L+1)$

$$
\hat{\breve{x}}(k \mid k-L+1)=A(k) e_{0}(k)
$$

Initial condition of $\hat{\breve{x}}(k \mid k-L+1)$ at $k=L: \hat{\breve{x}}(L \mid 1)$ 
Recursive equation for $e(k)$ :

$$
\begin{aligned}
& e(k)=e(k-1)+J(k)\left(\breve{y}(k)-\breve{H} A(k) e_{0}(k-1)\right) \\
& -J(k-L)\left(\breve{y}(k-L)-\breve{H} A(k-L) e_{0}(k-L-1)\right)
\end{aligned}
$$

Initial condition of $e(k)$ at $k=L: \bar{e}(L)$

Recursive equation for $e_{0}(k)$ :

$$
\begin{aligned}
& e_{0}(k)=e_{0}(k-1)+J_{0}(k)\left(\breve{y}(k)-\breve{H} A(k) e_{0}(k-1)\right) \\
& -J_{0}(k-L)\left(\breve{y}(k-L)-\breve{H} A(k-L) e_{0}(k-L-1)\right)
\end{aligned}
$$

Initial condition of $e_{0}(k)$ at $k=L: \bar{e}_{0}(L)$

Equation for $J(k)$ :

$$
J(k)=\left[\beta^{T}(k) \breve{H}^{T}-r(k-1) \alpha^{T}(k-1) \breve{\Phi}^{T} \breve{H}^{T}\right] \Lambda^{-1}(k)
$$

Equation for $J_{0}(k)$ :

$$
J_{0}(k)=\left[B^{T}(k) \breve{H}^{T}-r_{0}(k) A^{T}(k) \breve{\Phi}^{T} \breve{H}^{T}\right] \Lambda^{-1}(k)
$$

Recursive equation for $r_{0}(k)$ :

$$
\begin{aligned}
& r_{0}(k)=r_{0}(k-1)+J_{0}(k) \Lambda(k) J_{0}^{T}(k) \\
& -J_{0}(k-L) \Lambda(k-L) J_{0}^{T}(k-L)
\end{aligned}
$$

Initial condition of $r_{0}(k)$ at $k=L: \bar{r}_{0}(L)$

Equation for $\Lambda(k)$ :

$$
\Lambda(k)=\breve{H} \breve{K}(k, k) \breve{H}^{T}+R-\breve{H} A(k) r_{0}(k-1) A^{T}(k) \breve{H}^{T}
$$

Initial condition of FIR filtering estimate $\hat{x}(k \mid k-L+1)$ of $x(k)$ at $k=L: \hat{x}(L \mid 1)$

$$
\hat{x}(L \mid 1)=\alpha(L) \bar{e}(L)
$$

Recursive equation for $\bar{e}(L)$ :

$$
\begin{aligned}
& \bar{e}(L)=\bar{e}(L-1)+\bar{J}(L)\left(\breve{y}(L)-\breve{H} A(L) \bar{e}_{0}(L-1)\right), \\
& \bar{e}(0)=0
\end{aligned}
$$

Equation for $\bar{J}(L)$ :

$$
\bar{J}(L)=\left(\beta^{T}(L) \breve{H}^{T}-\bar{r}(L-1) \alpha^{T}(L-1) A^{T}(1) \breve{H}^{T}\right) \bar{\Lambda}^{-1}(L)
$$

Recursive equation for $\bar{r}(L)$ :

$$
\begin{aligned}
& \bar{r}(L)=\bar{r}(L-1)+\bar{J}(L) \bar{\Lambda}(L) \bar{J}^{T}(L), \\
& \bar{r}(0)=0
\end{aligned}
$$

Initial condition of FIR filtering estimate $\hat{\breve{x}}(k \mid k-L+1)$ of $\breve{x}(k)$ : at $k=L: \hat{x}(L \mid 1)$

$$
\hat{\breve{x}}(L \mid 1)=A(L) \bar{e}_{0}(k)
$$

Recursive equation for $\bar{e}_{0}(L)$ :

$$
\begin{aligned}
& \bar{e}_{0}(L)=\bar{e}_{0}(L-1)+\bar{J}_{0}(L)\left(\breve{y}(L)-\breve{H} A(L) \bar{e}_{0}(L-1)\right), \\
& \bar{e}_{0}(0)=0
\end{aligned}
$$

Equation for $\bar{J}_{0}(L)$ :

$$
\bar{J}_{0}(L)=\left(B^{T}(L) \breve{H}^{T}-\bar{r}_{0}(L-1) A^{T}(L) \breve{H}^{T}\right) \bar{\Lambda}^{-1}(L)
$$

Recursive equation for $\bar{r}_{0}(L)$ :

$$
\begin{aligned}
& \bar{r}_{0}(L)=\bar{r}_{0}(L-1)+\bar{J}_{0}(L) \bar{\Lambda}_{0}(L) \bar{J}_{0}^{T}(L), \\
& \bar{r}_{0}(0)=0
\end{aligned}
$$

Equation for $\bar{\Lambda}(L)$ :

$$
\bar{\Lambda}(L)=\breve{H} \breve{K}(L, L) \breve{H}^{T}+R-\breve{H} A(L) \bar{r}_{0}(L-1) A^{T}(L) \breve{H}^{T}
$$




\section{Proof}

By introducing an equation

$$
J(s) \Lambda(s)=\beta^{T}(s) \breve{H}^{T}-\sum_{i=s-1-L+1}^{s-1} J(i) \Lambda(i) g^{T}(s-1, i) \breve{\Phi}^{T} \breve{H}^{T}
$$

from (12) and (37), the optimal impulse response function $g(k, s)$ satisfies

$$
g(k, s)=\alpha(k) J(s) .
$$

By substituting (38) into (37), and introducing

$$
\begin{gathered}
r(k)=\sum_{i=k-L+1}^{k} J(i) \Lambda(i) J^{T}(i), \\
J(k) \Lambda(k)=\beta^{T}(k) \breve{H}^{T}-r(k-1) \alpha^{T}(k-1) \breve{\Phi}^{T} \breve{H}^{T}
\end{gathered}
$$

is obtained. By subtracting $r(k-1)$ from $r(k)$, it follows that

$$
\begin{aligned}
& r(k)-r(k-1)=J(k) \Lambda(k) J^{T}(k) \\
& -J(k-L) \Lambda(k-L) J^{T}(k-L), r(0)=0 .
\end{aligned}
$$

By substituting (38) into (11) and introducing $e(k)$, given by

$$
e(k)=\sum_{i=k-L+1}^{k} J(i) v(i)
$$

it follows that

$$
\begin{aligned}
& \hat{x}(k \mid k-L+1)=\alpha(k) \sum_{i=k-L+1}^{k} J(i) v(i), \\
& =\alpha(k) e(k) .
\end{aligned}
$$

By subtracting $e(k-1)$ from $e(k)$, it follows that

$$
\begin{aligned}
& e(k)-e(k-1)=J(k)(\breve{y}(k)-\breve{H} \breve{\Phi} \hat{\grave{x}}(k-1 \mid k-1-L+1)) \\
& -J(k-L)(\breve{y}(k-L)-\breve{H} \breve{\Phi} \hat{x}(k-L-1 \mid k-L-1-L+1)) .
\end{aligned}
$$

Let the FIR filtering estimate $\hat{x}(k \mid k-L+1)$ of $\breve{x}(k)$ be given by

$$
\begin{aligned}
& \hat{\grave{x}}(k \mid k-L+1)=\sum_{i=k-L+1}^{k} g_{0}(k, i) v(i), \\
& v(i)=\breve{y}(i)-\breve{H} \breve{\Phi} \hat{\grave{x}}(i-1 \mid i-1-L+1) .
\end{aligned}
$$

Likewise $g(k, s)$ in $(17)$, it is seen that $g_{0}(k, s)$ satisfies

$$
\begin{aligned}
& g_{0}(k, s) \Lambda_{0}(s)=E\left[\breve{x}(k) v^{T}(s)\right] \\
& =E\left[\breve{x}(k)(\breve{y}(s)-\breve{H} \breve{\Phi} \hat{\breve{x}}(s-1 \mid s-1-L+1))^{T}\right] \\
& =E\left[\breve{x}(k) \breve{y}^{T}(s)\right]-\sum_{i=s-L+1}^{s-1} E\left[\breve{x}(k) v^{T}(i)\right] g_{0}^{T}(s, i) \breve{\Phi}^{T} \breve{H}^{T} \\
& =A(k) B^{T}(s) \breve{H}^{T}-\sum_{i=s-L+1}^{s-1} g_{0}(k, i) \Lambda_{0}(i) g_{0}^{T}(s-1, i) \breve{\Phi}^{T} \breve{H}^{T} .
\end{aligned}
$$

By introducing

$$
J_{0}(s) \Lambda_{0}(s)=B^{T}(s) \breve{H}^{T}-\sum_{i=s-L+1}^{s-1} J_{0}(i) \Lambda_{0}(i) g_{0}^{T}(s-1, i) \breve{\Phi}^{T} \breve{H}^{T}
$$


$g_{0}(k, s)$ satisfies

$$
g_{0}(k, s)=A(k) J_{0}(s) .
$$

Also, by introducing

$$
r_{0}(k)=\sum_{i=k-L+1}^{k} J_{0}(i) \Lambda_{0}(i) J_{0}^{T}(i),
$$

(47) is rewritten as

$$
J_{0}(k) \Lambda_{0}(k)=B^{T}(k) \breve{H}^{T}-r_{0}(k) A^{T}(k) \breve{\Phi}^{T} \breve{H}^{T} .
$$

By subtracting $r_{0}(k-1)$ from $r_{0}(k)$, it follows that

$$
\begin{aligned}
& r_{0}(k)-r_{0}(k-1)=J_{0}(k) \Lambda_{0}(k) J_{0}^{T}(k) \\
& -J_{0}(k-L) \Lambda_{0}(k-L) J_{0}^{T}(k-L) .
\end{aligned}
$$

By introducing

$$
e_{0}(k)=\sum_{i=k-L+1}^{k} J_{0}(i) v(i),
$$

from (45), the FIR filtering estimate $\hat{\breve{x}}(k \mid k-L+1)$ of $\breve{x}(k)$ is given by

$$
\hat{\breve{x}}(k \mid k-L+1)=A(k) e_{0}(k) .
$$

By subtracting $e_{0}(k-1)$ from $e_{0}(k)$, it follows that

$$
\begin{aligned}
& e_{0}(k)-e_{0}(k-1)=J_{0}(k)\left(\breve{y}(k)-\breve{H} A(k) e_{0}(k-1)\right) \\
& -J_{0}(k-L)\left(\breve{y}(k-L)-\breve{H} A(k-L) e_{0}(k-L-1)\right), \\
& A(k) e_{0}(k-1)=\breve{\Phi} \hat{\grave{x}}(k-1 \mid k-1-L+1), \\
& A(k-L) e_{0}(k-L-1)=\breve{\Phi} \hat{\grave{x}}(k-L-1 \mid k-L-1-L+1) .
\end{aligned}
$$

From (45), (48) and (49), the variance $\Lambda(k)$ of the innovation process $v(k)$ is given by

$$
\begin{aligned}
& \Lambda(k)=E\left[v(k) v^{T}(k)\right] \\
& =E\left[(\breve{y}(k)-\breve{H} \breve{\Phi} \hat{x}(k-1 \mid k-1-L+1))(\breve{y}(k)-\breve{H} \breve{\Phi} \hat{x}(k-1 \mid k-1-L+1))^{T}\right] \\
& =\breve{H} \breve{K}(k, k) \breve{H}^{T}+R-\breve{H} \breve{\Phi} E\left[\hat{x}(k-1 \mid k-1-L+1) \hat{\breve{x}}^{T}(k-1 \mid k-1-L+1)\right] \\
& =\breve{H} \breve{K}(k, k) \breve{H}^{T}+R-\breve{H} A(k) r_{0}(k-1) A^{T}(k) \breve{H}^{T}
\end{aligned}
$$

Let the initial condition of the FIR filtering estimate of $x(k)$ at $k=L$ be $\hat{x}(L \mid 1)$.

$$
\begin{aligned}
& \hat{x}(L \mid 1)=\sum_{i=1}^{L} \bar{g}(L, i) \bar{v}(i), \\
& \bar{v}(i)=\breve{y}(i)-\breve{H} \breve{\Phi} \hat{x}(i-1 \mid 1)
\end{aligned}
$$

Let the variance of the innovation process $\bar{v}(L)$ be $\bar{\Lambda}(L)$.

$$
\begin{aligned}
& \bar{\Lambda}(L)=E\left[\bar{v}(L) \bar{v}^{T}(L)\right] \\
& =E\left[(\breve{y}(L)-\breve{H} \breve{\Phi} \hat{x}(L-1 \mid 1))(\breve{y}(k)-\breve{H} \breve{\Phi} \hat{x}(L-1 \mid 1))^{T}\right] \\
& =\breve{H} \breve{K}(L, L) \breve{H}^{T}+R-\breve{H} \breve{\Phi} E\left[\hat{x}(L-1 \mid 1) \hat{\breve{x}}^{T}(L-1 \mid 1)\right] \breve{\Phi}^{T} \breve{H}^{T} \\
& =\breve{H} \breve{K}(L, L) \breve{H}^{T}+R-\breve{H} A(L) \bar{r}_{0}(L-1) A^{T}(L) \breve{H}^{T}
\end{aligned}
$$

Here,

$$
\bar{r}_{0}(L)=\sum_{i=1}^{L} \bar{J}_{0}(i) \bar{\Lambda}(i) \bar{J}_{0}^{T}(i)
$$

is introduced. $\bar{g}(L, s)$ in (56) satisfies

$$
\begin{aligned}
& \bar{g}(L, s) \bar{\Lambda}(s)=K_{x \breve{x}}(L, s) \breve{H}^{T}-\sum_{i=1}^{s-1} \bar{g}(L, i) \bar{\Lambda}(i) \bar{g}^{T}(s-1, i) \breve{\Phi}^{T} \breve{H}^{T}, \\
& K_{x \breve{x}}(L, s) \breve{H}^{T}=K_{x \breve{z}}(L, s) .
\end{aligned}
$$


By introducing

$$
\bar{J}(s) \bar{\Lambda}(s)=\beta^{T}(s) \breve{H}^{T}-\sum_{i=1}^{s-1} \bar{J}(i) \bar{\Lambda}(i) \bar{g}^{T}(s-1, i) \breve{\Phi}^{T} \breve{H}^{T}
$$

$\bar{g}(L, s)$ is given by

$$
\bar{g}(L, s)=\alpha(L) \bar{J}(s)
$$

By introducing

$$
\bar{r}(L)=\sum_{i=1}^{L} \bar{J}(i) \bar{\Lambda}(i) \bar{J}^{T}(i)
$$

(60) is written as

$$
\begin{aligned}
& \bar{J}(L) \bar{\Lambda}(L)=\beta^{T}(L) \breve{H}^{T}-\bar{r}(L-1) \alpha^{T}(L-1) \breve{\Phi}^{T} \breve{H}^{T}, \\
& \breve{\Phi}^{T}=A^{T}(1) .
\end{aligned}
$$

By substituting (61) into (56) and introducing

$$
\begin{gathered}
\bar{e}(L)=\sum_{i=1}^{L} \bar{J}(i) \bar{v}(i), \\
\hat{x}(L \mid 1)=\alpha(L) \bar{e}(L)
\end{gathered}
$$

is obtained, By subtracting $\bar{r}(k-1)$ from $\bar{r}(k)$, it follows that

$$
\begin{aligned}
& \bar{r}(k)=\bar{r}(k-1)+\bar{J}(k) \bar{\Lambda}(k) \bar{J}^{T}(k), \\
& \bar{r}(0)=0 .
\end{aligned}
$$

By subtracting $\bar{e}(L-1)$ from $\bar{e}(L)$, it follows that

$$
\begin{aligned}
& \bar{e}(L)=\bar{e}(L-1)+\bar{J}(L)(\breve{y}(L)-\breve{H} \breve{\Phi} \hat{x}(L-1 \mid 1)), \\
& \bar{r}(0)=0 .
\end{aligned}
$$

Let the initial condition of the FIR filtering estimate of $\hat{x}(k)$ at $k=L$ be $\hat{x}(L \mid 1)$.

$$
\begin{aligned}
& \hat{\grave{x}}(L \mid 1)=\sum_{i=1}^{L} \bar{g}_{0}(L, i) \bar{v}(i), \\
& \bar{v}(k)=\breve{y}(k)-\breve{H} \breve{\Phi} \hat{x}(k-1 \mid 1)
\end{aligned}
$$

Here, $\bar{g}_{0}(L, s)$ satisfies

$$
\bar{g}_{0}(L, s) \bar{\Lambda}(s)=\breve{K}(L, s) \breve{H}^{T}-\sum_{i=1}^{s-1} \bar{g}_{0}(L, i) \bar{\Lambda}(i) \bar{g}_{0}^{T}(s-1, i) \breve{\Phi}^{T} \breve{H}^{T}
$$

By introducing

$$
\bar{J}_{0}(s) \bar{\Lambda}(s)=B^{T}(s) \breve{H}^{T}-\sum_{i=1}^{s-1} \bar{J}_{0}(i) \bar{\Lambda}(i) \bar{g}_{0}^{T}(s-1, i) \breve{\Phi}^{T} \breve{H}^{T}
$$

$\bar{g}_{0}(k, s)$ is given by

$$
\bar{g}_{0}(L, s)=A(L) \bar{J}_{0}(s)
$$

From (58),

$$
\bar{J}_{0}(L) \bar{\Lambda}(L)=B^{T}(L) \breve{H}^{T}-\bar{r}_{0}(L-1) A^{T}(L) \breve{H}^{T}
$$

is obtained. By subtracting $\bar{r}_{0}(L-1)$ from $\bar{r}_{0}(L)$, it follows that

$$
\begin{aligned}
& \bar{r}_{0}(L)=\bar{r}_{0}(L-1)+\bar{J}_{0}(L) \bar{\Lambda}(L) \bar{J}_{0}^{T}(L), \\
& \bar{r}_{0}(0)=0 .
\end{aligned}
$$


By substituting (71) into (68) and introducing

$$
\begin{gathered}
\bar{e}_{0}(L)=\sum_{i=1}^{L} \bar{J}_{0}(i) \bar{v}(i), \\
\hat{x}(L \mid 1)=A(L) \bar{e}_{0}(L)
\end{gathered}
$$

is obtained. By subtracting $\bar{e}_{0}(L-1)$ from $\bar{e}_{0}(L)$, it follows that

$$
\begin{aligned}
& \bar{e}_{0}(L)=\bar{e}_{0}(L-1)+\bar{J}_{0}(L)\left(\breve{y}(L)-\breve{H} A(L) \bar{e}_{0}(L-1)\right), \\
& A(L) \bar{e}_{0}(L-1)=\breve{\Phi} \hat{x}(L-1 \mid 1), \bar{e}_{0}(0)=0 .
\end{aligned}
$$

(Q.E.D.)

Based on the robust RLS FIR filter in Theorem 2, Theorem 3 proposes the robust RLS Wiener FIR algorithm for the filtering estimate of the signal $z(k)$.

Theorem 3 Let the state and the observation equations, including the uncertain quantities $\Delta \Phi$ and $\Delta H$ be given by (1). Let $\Phi$ and $H$ represent the system and observation matrices respectively for the signal $z(k)$. Let $\breve{\Phi}$ and $\breve{H}$ represent the system and observation matrices respectively for the degraded signal $\breve{z}(k)$, which is fitted to the AR model (3) of the order $N$. Let the variance $\breve{K}(k, k)$ of the state vector $\breve{x}(k)$ for the degraded signal $\breve{z}(k)$ and the cross-variance function $K_{x \breve{x}}(k, k)$ of the state vector $x(k)$ for the signal $z(k)$ with $\breve{x}(k)$ be given. Let the variance of the white observation noise $v(k)$ be $R$. Then, the robust RLS Wiener algorithm for the FIR filtering estimate $\hat{z}(k \mid k-L+1)$ of the signal $z(k)$ consists of (77)-(91) in linear discrete-time stochastic systems.

FIR filtering estimate of the signal $z(k): \hat{z}(k \mid k-L+1)$

$$
\hat{z}(k \mid k-L+1)=H \hat{x}(k \mid k-L+1)
$$

FIR filtering estimate of the state vector $x(k): \hat{x}(k \mid k-L+1)$

$$
\begin{aligned}
& \hat{x}(k \mid k-L+1)=\Phi \hat{x}(k-1 \mid k-1-L+1) \\
& +G(k)(\breve{y}(k)-\breve{H} \breve{\Phi} \hat{\breve{x}}(k-1 \mid k-1-L+1)) \\
& -\Phi^{L} G(k-L)(\breve{y}(k-L)-\breve{H} \breve{\Phi} \hat{\breve{x}}(k-L-1 \mid k-L-1-L+1))
\end{aligned}
$$

Initial condition of $\hat{x}(k \mid k-L+1)$ at $k=L: \hat{x}(L \mid 1)$

FIR filtering estimate of the state vector $\breve{x}(k): \hat{x}(k \mid k-L+1)$

$$
\begin{aligned}
& \hat{\breve{x}}(k \mid k-L+1)=\breve{\Phi} \hat{\grave{x}}(k-1 \mid k-1-L+1) \\
& +G_{0}(k)(\breve{y}(k)-\breve{H} \breve{\Phi} \hat{\grave{x}}(k-1 \mid k-1-L+1)) \\
& -\Phi^{L} G_{0}(k-L)(\breve{y}(k-L)-\breve{H} \breve{\Phi} \hat{\grave{x}}(k-L-1 \mid k-L-1-L+1))
\end{aligned}
$$

Initial condition of $\hat{\breve{x}}(k \mid k-L+1)$ at $k=L: \hat{\breve{x}}(L \mid 1)$

FIR filter gain for $\hat{x}(k \mid k-L+1): G(k)$

$$
G(k)=\left[K_{x \breve{x}}(k, k) \breve{H}^{T}-\Phi S(k-1) \breve{\Phi}^{T} \breve{H}^{T}\right] \Lambda^{-1}(k)
$$

FIR filter gain for $\hat{\breve{x}}(k \mid k-L+1): G_{0}(k)$

$$
G_{0}(k)=\left[\breve{K}(k, k) \breve{H}^{T}-\breve{\Phi} S_{0}(k-1) \breve{\Phi}^{T} \breve{H}^{T}\right] \Lambda^{-1}(k)
$$

Equation for $\Lambda(k)$ :

$$
\Lambda(k)=R+\breve{H} \breve{K}(k, k) \breve{H}^{T}-\breve{H} \breve{\Phi} S_{0}(k-1) \breve{\Phi}^{T} \breve{H}^{T}
$$


Recursive equation for $S(k)$ :

$$
\begin{aligned}
& S(k)=\Phi S(k-1) \breve{\Phi}^{T}+G(k) \Lambda(k) G^{T}(k) \\
& -\Phi^{L} G(k-L) \Lambda(k-L) G^{T}(k-L)\left(\Phi^{T}\right)^{L}
\end{aligned}
$$

Initial condition of $S(k)$ at $k=L: \bar{S}(L)$

Recursive equation for $S_{0}(k)$ :

$$
\begin{aligned}
& S_{0}(k)=\breve{\Phi} S_{0}(k-1) \breve{\Phi}^{T}+G_{0}(k) \Lambda(k) G_{0}^{T}(k) \\
& -\breve{\Phi}^{L} G_{0}(k-L) \Lambda(k-L) G_{0}^{T}(k-L)\left(\breve{\Phi}^{T}\right)^{L}
\end{aligned}
$$

Initial condition of $S_{0}(k)$ at $k=L: \bar{S}_{0}(L)$

Recursive equation for $\hat{x}(L \mid 1)$ :

$$
\begin{aligned}
& \hat{x}(L \mid 1)=\Phi \hat{x}(L-1 \mid 1)+\bar{G}(L)(\breve{y}(L)-\breve{H} \breve{\Phi} \hat{x}(L-1 \mid 1)), \\
& \hat{x}(0 \mid 1)=0
\end{aligned}
$$

Filter gain for $\hat{x}(L \mid 1)$ in $(85): \bar{G}(L)$

$$
\begin{aligned}
& \bar{G}(L)=\left[K_{x \breve{z}}(L, L)-\Phi \bar{S}(L-1) \breve{\Phi}^{T} \breve{H}^{T}\right] \bar{\Lambda}^{-1}(L), \\
& K_{x \breve{z}}(L, L)=K_{x \breve{x}}(L, L) \breve{H}^{T}
\end{aligned}
$$

Recursive equation for $\hat{\breve{x}}(L \mid 1)$ :

$$
\begin{aligned}
& \hat{\grave{x}}(L \mid 1)=\breve{\Phi} \hat{\breve{x}}(L-1 \mid 1)+\bar{G}_{0}(L)(\breve{y}(L)-\breve{H} \breve{\Phi} \hat{\grave{x}}(L-1 \mid 1)), \\
& \hat{\breve{x}}(0 \mid 1)=0
\end{aligned}
$$

Filter gain for $\hat{x}(L \mid 1)$ in $(87): \bar{G}_{0}(L)$

$$
\bar{G}_{0}(L)=\left[\breve{K}(L, L) \breve{H}^{T}-\breve{\Phi} \bar{S}_{0}(L-1) \breve{\Phi}^{T} \breve{H}^{T}\right] \bar{\Lambda}^{-1}(L)
$$

Equation for $\bar{\Lambda}(L)$ :

$$
\bar{\Lambda}(L)=R+\breve{H} \breve{K}(L, L) \breve{H}^{T}-\breve{H} \breve{\Phi} \bar{S}_{0}(L-1) \breve{\Phi}^{T} \breve{H}^{T}
$$

Recursive equation for $\bar{S}(L)$ :

$$
\begin{aligned}
& \bar{S}(L)=\Phi \bar{S}(L-1) \breve{\Phi}^{T}+\bar{G}(L) \bar{\Lambda}(L) \bar{G}^{T}(L), \\
& \bar{S}(0)=0
\end{aligned}
$$

Recursive equation for $\bar{S}_{0}(L)$ :

$$
\begin{aligned}
& \bar{S}_{0}(L)=\breve{\Phi} \bar{S}_{0}(L-1) \breve{\Phi}^{T}+\bar{G}_{0}(L) \bar{\Lambda}(L) \bar{G}_{0}^{T}(L), \\
& \bar{S}_{0}(0)=0
\end{aligned}
$$

\section{Proof}

By substituting (44) into (43) and introducing

$$
G(k)=\alpha(k) J(k),
$$

(78) is obtained. By substituting (54) into (53) and introducing

$$
G_{0}(k)=A(k) J_{0}(k),
$$

(79) is obtained. By substituting (40) into (92), using (10) and introducing

$$
S(k)=\alpha(k) r(k) \alpha^{T}(k),
$$


(80) is obtained. By substituting (50) into (93), using (6) and introducing

$$
S_{0}(k)=A(k) r_{0}(k) A^{T}(k),
$$

(81) is obtained. By substituting (95) into (55) and using $A(k)=\breve{\Phi}^{k},(82)$ is obtained. By substituting

(41) into (94) and using (92) with $\alpha(k)=\Phi^{k},(83)$ is obtained. By substituting (51) into (95) and using

(93) with $A(k)=\breve{\Phi}^{k},(84)$ is obtained.

By substituting (67) into (65) and introducing

$$
\bar{G}(L)=\alpha(L) \bar{J}(L),
$$

(85) is obtained. By substituting (63) into (96), using (10) and introducing

$$
\bar{S}(L)=\alpha(L) \bar{r}(L) \alpha^{T}(L),
$$

(86) is obtained. By substituting (76) into (75) and introducing

$$
\bar{G}_{0}(L)=A(L) \bar{J}_{0}(L),
$$

(87) is obtained. By substituting (72) into (98) and introducing

$$
\bar{S}_{0}(L)=A(L) \bar{r}_{0}(L) A^{T}(L),
$$

(88) is obtained. By substituting (99) into (57) and using $A(k)=\breve{\Phi}^{k},(89)$ is obtained. By substituting (66) into (97) and using (96), (90) is obtained. By substituting (73) into (99) and using (98), (91) is obtained.

(Q.E.D.)

Concerning the stability of the robust RLS Wiener FIR filtering algorithm, the following conditions are required.

1. All the real parts in the eigenvalues of the matrix $\Phi$ are negative.

2. All the real parts in the eigenvalues of the matrix $\breve{\Phi}-G_{0}(k) \breve{H} \breve{\Phi}$ are negative.

3. $R+\breve{H}\left[\breve{K}(k, k)-\breve{\Phi} S_{0}(k-1) \breve{\Phi}^{T}\right] \breve{H}^{T}>0$

4. All the real parts in the eigenvalues of the matrix $\breve{\Phi}-\bar{G}_{0}(k) \breve{H} \breve{\Phi}$ are negative.

5. $R+\breve{H}\left[\breve{K}(k, k)-\breve{\Phi} \bar{S}_{0}(k-1) \breve{\Phi}^{T}\right] \breve{H}^{T}>0$

In section 4 , the existence of the robust RLS Wiener FIR filtering estimate $\hat{z}(k \mid k-L+1)$ of the signal $z(k)$ is shown.

\section{Filtering Error Variance Function of Signal}

Let the variance function of the FIR filtering error $z(k)-\hat{z}(k \mid k-L+1)$ be denoted by $\tilde{P}_{z}(k)$. Let the auto-covariance function $K(k, s)$ of the state vector $x(k)$ be given by

$$
\begin{aligned}
& K(k, s)=\left\{\begin{array}{l}
A_{x}(k) B_{x}^{T}(s), 0 \leq s \leq k, \\
B_{x}(k) A_{x}^{T}(s), 0 \leq k \leq s,
\end{array}\right. \\
& A_{x}(k)=\alpha(k)=\Phi^{k}, B_{x}^{T}(s)=\Phi^{-s} K(s, s) .
\end{aligned}
$$

Then the FIR filtering error variance function $\tilde{P}_{z}(k)$ is formulated as

$$
\begin{aligned}
& \tilde{P}_{z}(k)=H\left[K(k, k)-E\left[\hat{x}(k \mid k-L+1) \hat{x}^{T}(k \mid k-L+1)\right] H^{T}\right. \\
& =H\left[K(k, k)-E\left[x(k) \hat{x}^{T}(k \mid k-L+1)\right] H^{T} .\right.
\end{aligned}
$$

By using (39), (43) and (94), (101) is rewritten as

$$
\begin{aligned}
& \tilde{P}_{z}(k)=H\left(K(k, k)-\alpha(k) r(k)\left(\alpha^{T}\right)^{k}\right) H^{T} \\
& =H(K(k, k)-S(k)) H^{T}
\end{aligned}
$$


Here, the RLS Wiener FIR filtering error variance function $\tilde{P}_{z}(k)$ is calculated by (102) together with $(80) \sim(84)$ recursively. Since $\tilde{P}_{z}(k)$ is the semi-definite function, the RLS Wiener FIR filtering variance function of the signal $z(k), H E\left[\hat{x}(k \mid k-L+1) \hat{x}^{T}(k \mid k-L+1)\right] H^{T}$, is upper bounded by $H K(k, k) H^{T}$ and lower bounded by the zero matrix as

$$
0 \leq H E\left[\hat{x}(k \mid k-L+1) \hat{x}^{T}(k \mid k-L+1)\right] H^{T} \leq H K(k, k) H^{T} .
$$

This validates the existence of the robust RLS Wiener FIR filtering estimate $\hat{z}(k \mid k-L+1)$ of the signal $z(k)$.

\section{A Numerical Simulation Example}

Let a scalar observation equation and the state equation for $x(k)$ be described by

$$
\begin{aligned}
& y(k)=z(k)+v(k), z(k)=H x(k), H=\left[\begin{array}{ll}
1 & 0
\end{array}\right], x(k)=\left[\begin{array}{l}
x_{1}(k) \\
x_{2}(k)
\end{array}\right], \\
& x(k+1)=\Phi x(k)+\Gamma w(k), \Phi=\left[\begin{array}{cc}
0 & 1 \\
-a_{2}-a_{1}
\end{array}\right], a_{1}=-0.1, a_{2}=-0.8, \Gamma=\left[\begin{array}{l}
0 \\
1
\end{array}\right], \\
& E[v(k) v(s)]=R \delta_{K}(k-s), E[w(k) w(s)]=Q \delta_{K}(k-s), Q=0.5^{2} .
\end{aligned}
$$

It should be noted that the signal $z(k)$ is generated by the second-order AR model. The purpose of the simulation example is to estimate the signal $z(k)$ in terms of the observed value $\breve{y}(k)$, generated by the state-space model (105) with the uncertain quantities $\Delta H(k)$ and $\Delta \Phi(k)$.

$$
\begin{aligned}
& \breve{y}(k)=\breve{z}(k)+v(k), \breve{z}(k)=\bar{H}(k) \bar{x}(k), \bar{x}(k)=\left[\begin{array}{c}
\bar{x}_{1}(k) \\
\bar{x}_{2}(k)
\end{array}\right], \\
& \bar{H}(k)=H+\Delta H(k)=\left[1+\Delta_{3}(k) 0\right], \Delta H(k)=\left[\Delta_{3}(k) 0\right], \Delta_{3}(k)=0.1, \\
& \bar{x}(k+1)=\bar{\Phi}(k) \bar{x}(k)+\Gamma w(k), \bar{\Phi}(k)=\Phi+\Delta \Phi(k), \Delta \Phi(k)=\left[\begin{array}{cc}
0 & 0 \\
\Delta_{2}(k) & \Delta_{1}(k)
\end{array}\right], \\
& \Delta_{1}(k)=0.01, \Delta_{2}(k)=-0.1
\end{aligned}
$$

Without using a priori knowledge on $\Delta H(k)$ and $\Delta \Phi(k)$, the robust RLS Wiener FIR filter estimates the signal $z(k)$ recursively. In particular, the scalar degraded signal $\breve{z}(k)$ is fitted to the AR model of the $N$-th order.

$$
\begin{aligned}
& \breve{z}(k)=-\breve{a}_{1} \breve{z}(k-1)-\breve{a}_{2} \breve{z}(k-2)-\cdots-\breve{a}_{N} \breve{z}(k-N)+\breve{e}(k), \\
& E[\breve{e}(k) \breve{e}(s)]=\breve{Q} \delta_{K}(k-s)
\end{aligned}
$$

From (4) and (105), $\breve{z}(k)$ is expressed as (107) with the 1 by $N$ observation vector $\breve{H}$.

$$
\breve{z}(k)=\breve{H} \breve{x}(k), \breve{H}=\left[\begin{array}{llllll}
1 & 0 & 0 & \cdots & 0 & 0
\end{array}\right] .
$$

In the simulation example, the state equation for $\breve{x}(k)$ in $(5)$ corresponds to the case of $m=1 . \breve{K}(k, s)=$ $\breve{K}(k-s)$ represents the auto-covariance function of the state vector $\breve{x}(k)$ in wide-sense stationary stochastic systems. $\breve{K}(k, s)$ is expressed in the form of the semi-degenerate function (6). $\breve{\Phi}$ in (6) represents the system matrix for the state vector $\breve{x}(k)$. Also, from the auto-covariance function $K_{\breve{z}}(k-s)=K_{\breve{z}}(s-k)=$ $E[\breve{z}(k) \breve{z}(s)]$ of the degraded signal $\breve{z}(k)$, the auto-variance function $\breve{K}(k, k)$ of the state vector $\breve{x}(k)$ is expressed as

$$
\begin{aligned}
& \breve{K}(k, k)=E\left[\left[\begin{array}{c}
\breve{z}(k) \\
\breve{z}(k+1) \\
\vdots \\
\breve{z}(k+N-2) \\
\breve{z}(k+N-1)
\end{array}\right]\right] \\
& \times[\breve{z}(k) \breve{z}(k+1) \cdots \breve{z}(k+N-2) \breve{z}(k+N-1)] \\
& =\left[\begin{array}{ccccc}
K_{\breve{z}}(0) & K_{\breve{z}}(1) & \cdots & K_{\breve{z}}(N-2) & K_{\breve{z}}(N-1) \\
K_{\breve{z}}(1) & K_{\breve{z}}(0) & \cdots & K_{\breve{z}}(N-3) & K_{\breve{z}}(N-2) \\
\vdots & \vdots & \ddots & \vdots & \vdots \\
K_{\breve{z}}(N-2) & K_{\breve{z}}(N-3) & \cdots & K_{\breve{z}}(0) & K_{\breve{z}}(1) \\
K_{\breve{z}}(N-1) & K_{\breve{z}}(N-2) & \cdots & K_{\breve{z}}(1) & K_{\breve{z}}(0)
\end{array}\right] .
\end{aligned}
$$


Let $K_{z \breve{z}}(k, s)=E[z(k) \breve{z}(s)]$ represent the cross-covariance function of the signal $z(k)$ with the degraded signal $\breve{z}(s)$. From (4) and (104), the cross-covariance function $K_{x \breve{x}}(k, s)$ is expressed as

$$
\begin{aligned}
& K_{x \breve{x}}(k, s)=\Phi^{k-s} K_{x \breve{x}}(s, s), 0 \leq s \leq k, \\
& K_{x \breve{x}}(k, k)=E\left[\left[\begin{array}{l}
x_{1}(k) \\
x_{2}(k)
\end{array}\right][\breve{z}(k) \breve{z}(k+1) \cdots \breve{z}(k+N-2) \breve{z}(k+N-1)]\right] \\
& =\left[\begin{array}{lllll}
E\left[x_{1}(k) \breve{z}(k)\right] & E\left[x_{1}(k) \breve{z}(k+1)\right. & \cdots & E\left[x_{1}(k) \breve{z}(k+N-2)\right] & E\left[x_{1}(k) \breve{z}(k+N-1)\right] \\
E\left[x_{2}(k) \breve{z}(k)\right] & E\left[x_{2}(k) \breve{z}(k+1)\right. & \cdots & E\left[x_{2}(k) \breve{z}(k+N-2)\right] & E\left[x_{2}(k) \breve{z}(k+N-1)\right]
\end{array}\right] \\
& =\left[\begin{array}{cc}
E[z(k) \breve{z}(k)] & E[z(k) \breve{z}(k+1) \\
E[z(k+1) \breve{z}(k)] & E[z(k+1) \breve{z}(k+1)
\end{array}\right. \\
& \cdots \quad E[z(k) \breve{z}(k+N-2)] \quad E[z(k) \breve{z}(k+N-1)] \\
& \cdots E[z(k+1) \breve{z}(k+N-2)] E[z(k+1) \breve{z}(k+N-1)]] \\
& =\left[\begin{array}{ccccc}
K_{z \breve{z}}(k, k) & K_{z \breve{z}}(k, k+1) & \cdots & K_{z \breve{z}}(k, k+N-2) & K_{z \breve{z}}(k, k+N-1) \\
K_{z \breve{z}}(k+1, k) & K_{z \breve{z}}(k+1, k+1) & \cdots & K_{z \breve{z}}(k+1, k+N-2) & K_{z \breve{z}}(k+1, k+N-1)
\end{array}\right] .
\end{aligned}
$$

The AR parameters $\breve{a}_{1}, \breve{a}_{2}, \cdots, \breve{a}_{N-1}, \breve{a}_{N}$ in (106) are calculated by

$$
\left[\begin{array}{ccccc}
K_{\breve{z}(0)} & K_{\breve{z}(1)} & \cdots & K_{\breve{z}}(N-2) & K_{\breve{z}}(N-1) \\
K_{\breve{z}(1)} & K_{\breve{z}(0)} & \cdots & K_{\breve{z}}(N-3) & K_{\breve{z}}(N-2) \\
\vdots & \vdots & \ddots & \vdots & \vdots \\
K_{\breve{z}}(N-2) & K_{\breve{z}}(N-3) & \cdots & K_{\breve{z}(0)} & K_{\breve{z}(1)} \\
K_{\breve{z}}(N-1) & K_{\breve{z}}(N-2) & \cdots & K_{\breve{z}}(1) & K_{\breve{z}}(0)
\end{array}\right]\left[\begin{array}{c}
\breve{a}_{1} \\
\breve{a}_{2} \\
\vdots \\
\breve{a}_{N-1} \\
\breve{a}_{N}
\end{array}\right]=-\left[\begin{array}{c}
K_{\breve{z}}(1) \\
K_{\breve{z}}(2) \\
\vdots \\
K_{\breve{z}}(N-1) \\
K_{\breve{z}}(N)
\end{array}\right]
$$

By substituting $H, \breve{H}, \Phi, \breve{\Phi}, K_{x \breve{x}}(k, k), \breve{K}(k, k)=\breve{K}(L, L)$ and $R$ into the robust RLS Wiener FIR filtering algorithm of Theorem 3, the filtering estimates are calculated recursively. In evaluating $\breve{\Phi}$ in $(7)$, $\breve{K}(k, k)$ in (108) and $K_{x \breve{x}}(k, k)$ in (109), the 2,000 number of signal and degraded signal data are used. Fig. 1 illustrates the signal $z(k)$ and its degraded signal $\breve{z}(k)$ caused by the uncertain parameters in the observation vector and the system matrix in (105) vs. $k, 1 \leq k \leq 500$, for the white Gaussian observation noise $N\left(0,0.3^{2}\right)$. Fig. 2 illustrates the signal $z(k)$ and the robust RLS Wiener filtering estimate $\hat{z}(k \mid 1)$ vs. $k, 1 \leq k \leq 200$, for the white Gaussian observation noise $N\left(0,0.3^{2}\right)$ in the case of the AR model order $N=5$. Here, $\hat{z}(k \mid 1)$ is computed by (85)-(91) recursively. It is noted that (85)-(91) constitute the robust RLS Wiener filtering algorithm in [8]. Fig. 3 illustrates the signal $z(k)$ and the RLS Wiener FIR filtering estimate $\hat{z}(k \mid k-L+1), L=200$, vs. $k, 201 \leq k \leq 500$, for the white Gaussian observation noise $N\left(0,0.3^{2}\right)$ in the case of the AR model order $N=5$. Fig. 4 compares the MSVs of the FIR filtering errors $z(k)-\hat{z}(k \mid k-L+1), L \leq k \leq 1000+L$, by the robust RLS Wiener FIR filter in Theorem 3 with the robust RLS Wiener filter [8], the robust Kalman filter [9] and the RLS Wiener FIR filter [10] vs. the finite interval $L$ for the white Gaussian observation noises $N\left(0,0.1^{2}\right), N\left(0,0.3^{2}\right), N\left(0,0.5^{2}\right)$ and $N\left(0,0.7^{2}\right)$. In the robust RLS Wiener FIR filter and the robust RLS Wiener filter [8], the AR model order is $N=5$. Here, the MSVs by the robust RLS Wiener filter [8] and the robust Kalman filter are calculated with the filtering errors $z(k)-\hat{z}(k \mid k), L \leq k \leq 1000+L$. From Fig. 4, the proposed robust RLS Wiener FIR filter is preferable in estimation accuracy to the robust Kalman filter [9] and the RLS Wiener FIR filter [10]. As the finite interval $L$ increases, the MSV of the filtering errors by the robust RLS Wiener filter FIR decreases and approaches the MSV by the robust RLS Wiener filter [8] for each observation noise. This indicates, with the finite number of observations, that the robust RLS Wiener FIR filter in Theorem 3 could attain almost the same estimation accuracy with the robust RLS Wiener filter [8]. Fig. 5 illustrates the MSVs of the FIR filtering errors $z(k)-\hat{z}(k \mid k-L+1), L \leq k \leq 1000+L$, by the robust RLS Wiener FIR filter in Theorem 3 vs. the finite interval $L$ for the white Gaussian observation noises $N\left(0,0.1^{2}\right)$, $N\left(0,0.3^{2}\right), N\left(0,0.5^{2}\right)$ and $N\left(0,0.7^{2}\right)$ in the case of the AR model order $N=10$. From Fig. 4 and Fig. 5 , the MSVs of the RLS Wiener FIR filtering errors for the AR model order $N=5$ are slightly smaller than those for the AR model order $N=10$. Here, the MSVs of the FIR filtering errors are evaluated by $\sum_{k=L}^{1000+L}(z(k)-\hat{z}(k \mid k-L+1))^{2} / 1001$. 


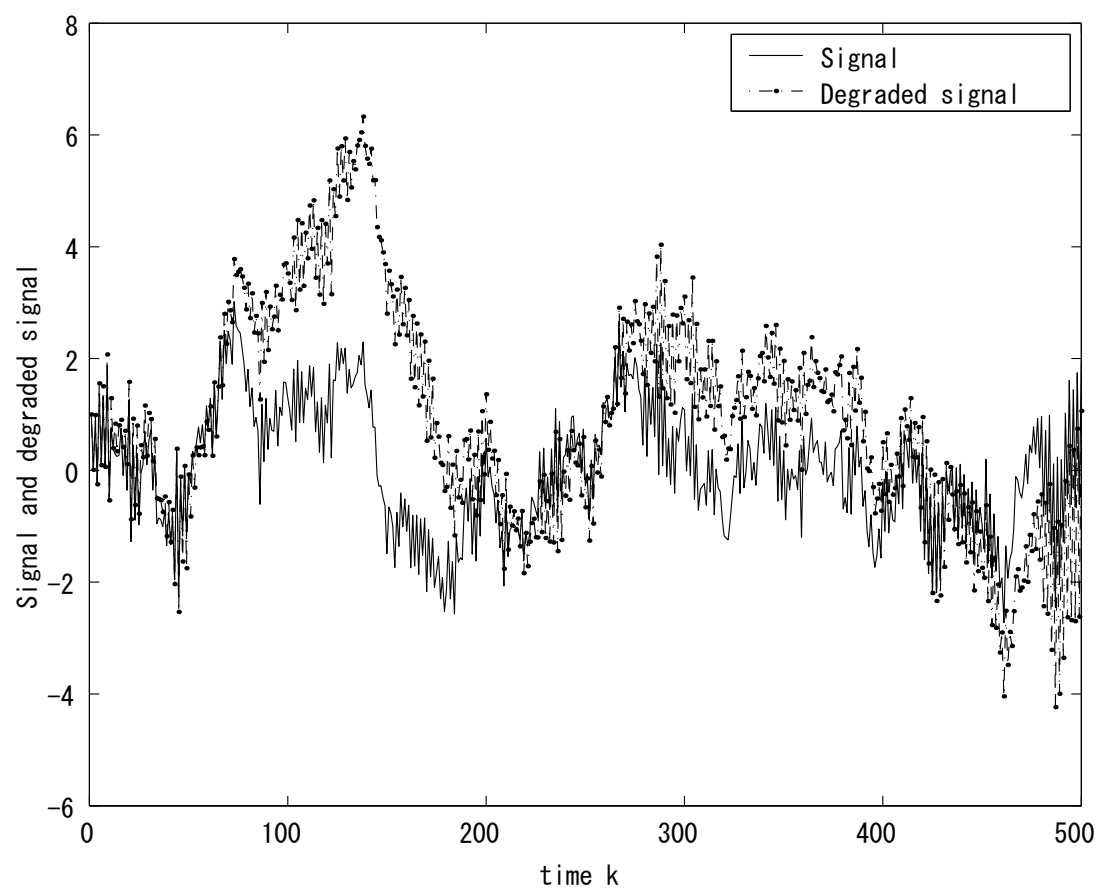

Figure 1. Signal $z(k)$ and degraded signal $\breve{z}(k)$ caused by the uncertain parameters for the white Gaussian observation noise $N\left(0,0.3^{2}\right)$.

\section{Conclusions}

This paper, in Theorem 2, has proposed the robust RLS FIR filter using the covariance information of the state vector for the degraded signal, the cross-covariance information of the state vector for the signal with the state vector for the degraded signal, the observation matrices for the signal and the degraded signal, and the variance of the white observation noise. Here, it is assumed that the signal and the degraded signal are fitted to the finite-order AR models. In Theorem 3 the robust RLS Wiener FIR filter has been proposed. The robust RLS Wiener FIR filtering algorithm uses the system and observation matrices for the signal and the degraded signal, the variance of the state vector for the degraded signal, the cross-variance function of the state vector for the signal with the state vector for the degraded signal, and the variance of the white observation noise.

In the numerical simulation example, for the signal process expressed by the second-order AR model, the robust RLS Wiener FIR filter in this paper is superior in estimation accuracy to the robust Kalman filter [9] and the RLS Wiener FIR filter [10]. As the finite interval $L$ increases, the MSV of the filtering errors by the robust RLS Wiener FIR filter decreases and approaches the MSV by the robust RLS Wiener filter [8] for each observation noise. This indicates, with the finite number of degraded observations, that the robust RLS Wiener FIR filter in Theorem 3 could attains almost the same estimation accuracy with the robust RLS Wiener filter [8].

\section{References}

1. A. H. Jazwinski, Limited memory optimal filtering. IEEE Trans. Automatic Control, vol.13, no.10, pp.558-563, 1968.

2. W. H. Kwon and S. Han, Receding Horizon Control: Model Predictive Control for State Models, London, UK: Springer; 2005.

3. S. Zhao S, Y. S. Shmaliy, P. Shi and C. K. Ahn, Fusion Kalman/UFIR filter for state estimation with uncertain parameters and noise statistics. IEEE Trans. Industrial Electronics, vol.64, no.4, pp.3075-3083, 2017. 


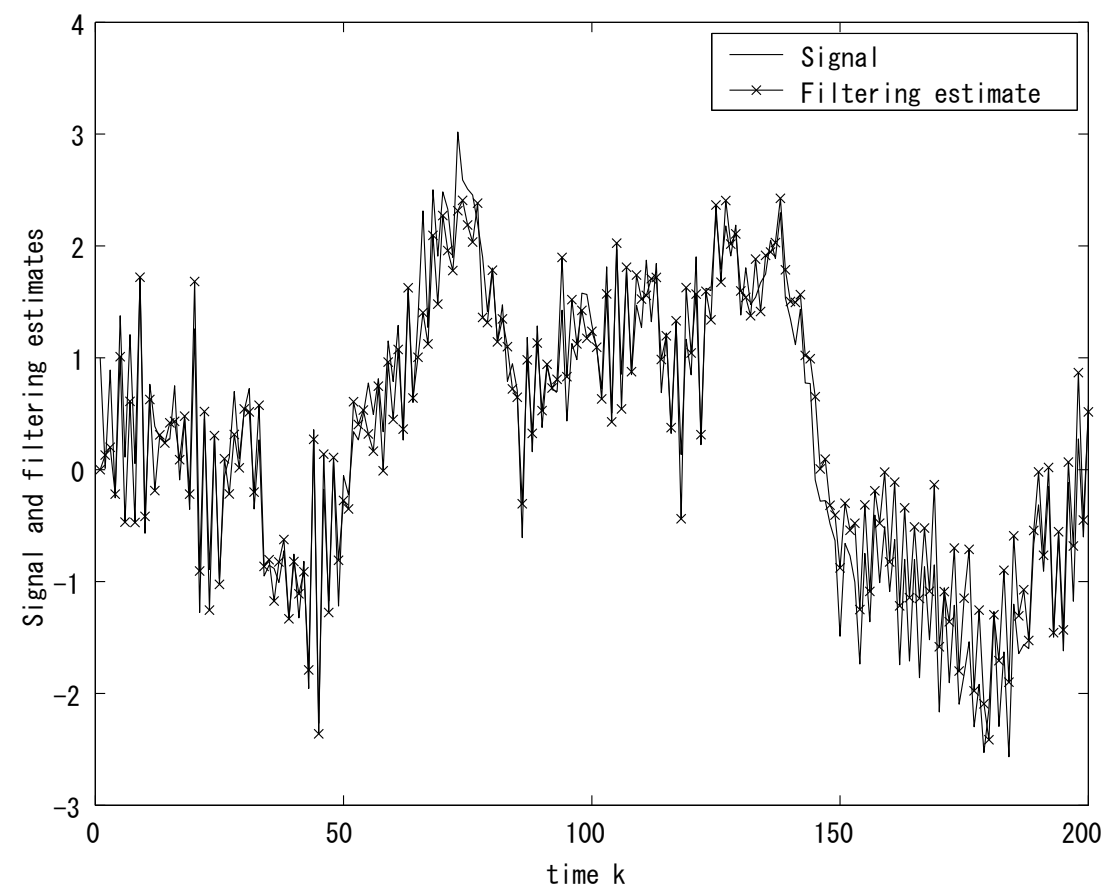

Figure 2. Signal $z(k)$ and filtering estimate $\hat{z}(k \mid 1)$ of the signal $z(k)$ vs. $k, 1 \leq k \leq 200$, for the white Gaussian observation noise $N\left(0,0.3^{2}\right)$ in the case of the AR model order $N=5$.

4. S. Zhao, Y. S. Shmaliy and F. Liu, Fast Kalman-like optimal unbiased FIR filtering with applications, IEEE Trans. Signal Processing, vol.64, no.9, pp.2284-97, 2016.

5. S. Zhao, Y. S. Shmaliy and F. Liu, Fast Kalman-like optimal FIR filter for time-variant systems with improved robustness, ISA Transactions, Volume 80, pp.160-168, 2018.

6. J. M. Pak, C. K. Ahn, Y. H. Mo and M. T. Lim, Moon Kyou Song, Maximum likelihood FIR filter for visual object tracking, Neurocomputing, 216, pp.543-553, 2016.

7. X. Wang, W. Liu and Z. Deng, Robust weighted fusion Kalman estimators for systems with multiplicative noises, missing measurements and uncertain-variance linearly correlated white noises, Aerospace Science and Technology, vol.68, pp.331-344, 2017.

8. S. Nakamori, Robust RLS Wiener signal estimators for discrete-time stochastic systems with uncertain parameters, Frontiers in Signal Processing, vol.1, no.3, pp.1-18, 2019.

9. X. Zhu, Y. C. Soh and L. Xie, Design and analysis of discrete-time robust Kalman filters, Automatica, vol.38, no.6, pp.1069-1077, 2002.

10. S. Nakamori, RLS Wiener FIR predictor and filter based on innovation approach in linear discrete-time stochastic systems, Frontiers in Signal Processing, vol.1, no.2, pp.49-61, 2017.

11. A. P. Sage and J. L. Melsa, Estimation Theory with Applications to Communications and Control, New York: McGraw-Hill, 1971. 


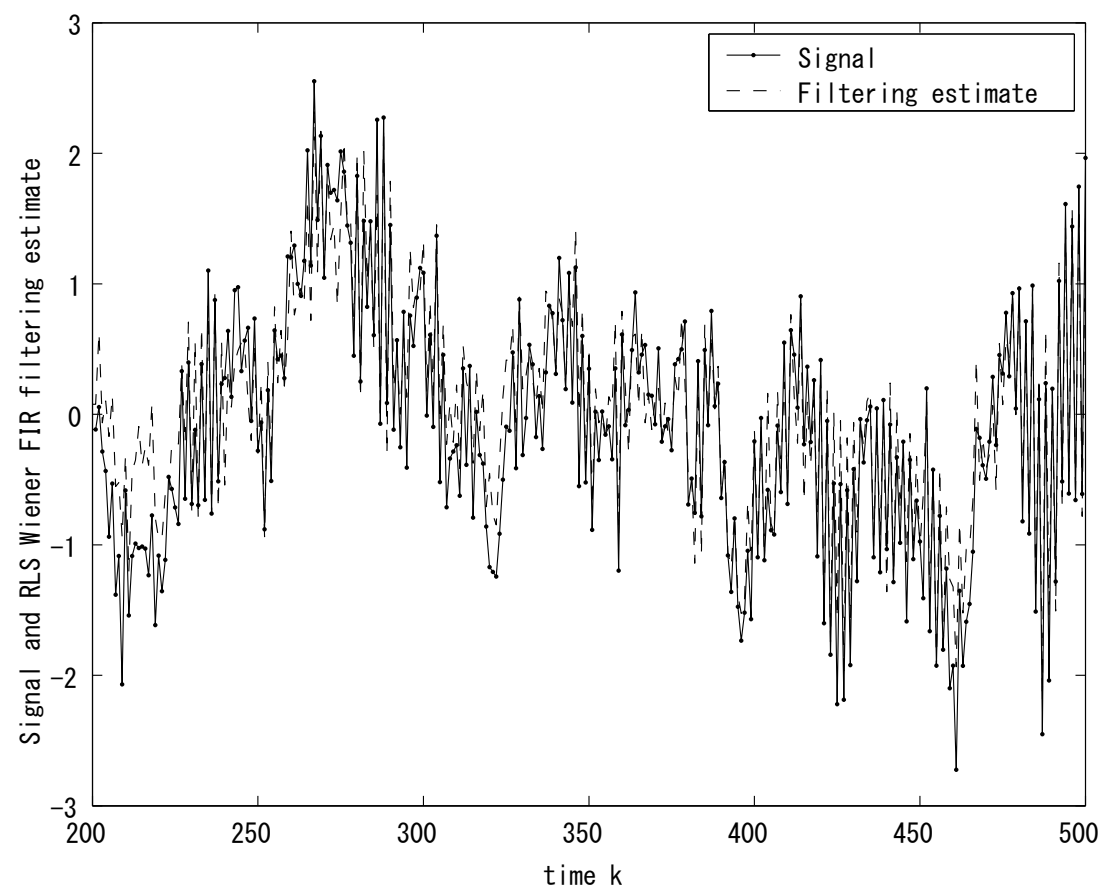

Figure 3. Signal $z(k)$ and RLS Wiener FIR filtering estimate $\hat{z}(k \mid k-L+1)$ of the signal $z(k)$ vs. $k, 201 \leq k \leq 500$, for the fixed interval $L=200$ and the white Gaussian observation noise $N\left(0,0.3^{2}\right)$ in the case of the AR model order $N=5$.

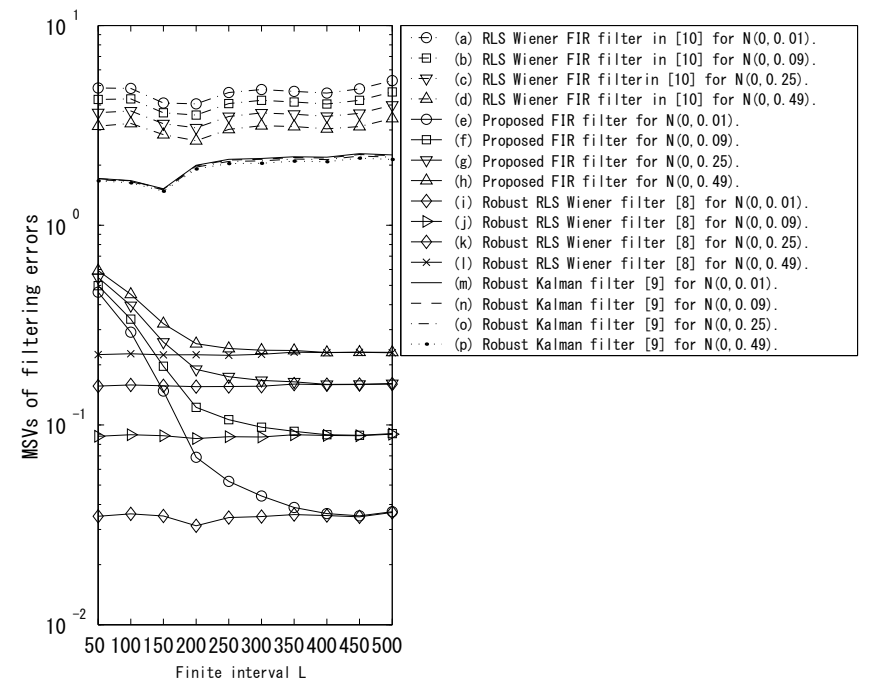

Figure 4. Comparison of MSV of the FIR filtering errors $z(k)-\hat{z}(k \mid k-L+1), L \leq k \leq 100+L$, by the robust RLS Wiener FIR filter in Theorem 3, with MSVs of filtering errors by the robust RLS Wiener filter [8], the robust Kalman filter [9] and the RLS Wiener FIR filter [10] vs. the finite interval $L$ for the white Gaussian observation noises $N\left(0,0.1^{2}\right), N\left(0,0.3^{2}\right), N\left(0,0.5^{2}\right)$ and $N\left(0,0.7^{2}\right)$. 


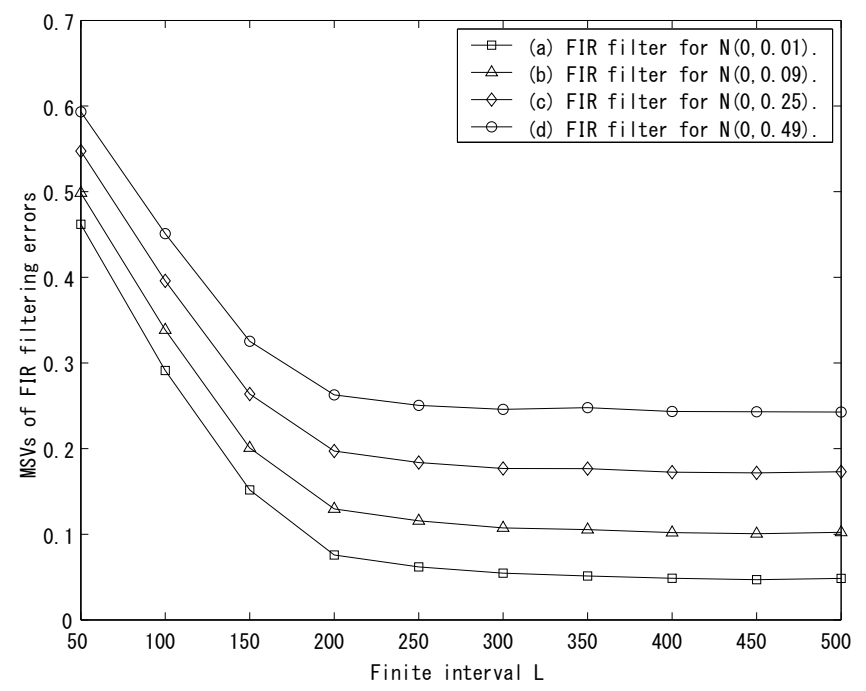

Figure 5. MSVs of the FIR filtering errors $z(k)-\hat{z}(k \mid k-L+1), L \leq k \leq 100+L$, by the robust RLS Wiener FIR filter, proposed in this paper, vs. the finite interval $L$ for the white Gaussian observation noises $N\left(0,0.1^{2}\right)$, $N\left(0,0.3^{2}\right), N\left(0,0.5^{2}\right)$ and $N\left(0,0.7^{2}\right)$ in the case of the AR model order $N=10$. 